Portland State University

PDXScholar

Psychology Faculty Publications and

Presentations

Psychology

$10-1-2017$

\title{
Proximal Predictors of Alcohol Use Among Japanese College Students
}

\author{
Staci Wendt \\ Human and Health Development Program, WestEd \\ Cynthia Mohr \\ Portland State University, cynthia.mohr@pdx.edu \\ Mo Wang \\ University of Florida \\ Sarah Haverly \\ Portland State University
}

Follow this and additional works at: https://pdxscholar.library.pdx.edu/psy_fac

Part of the Psychology Commons

Let us know how access to this document benefits you.

\section{Citation Details}

Wendt, S., Mohr, C., Wang, M., and Haverly, S. 2017. Proximal Predictors of Alcohol Use Among Japanese College Students. Substance Use \& Misuse.

This Post-Print is brought to you for free and open access. It has been accepted for inclusion in Psychology Faculty Publications and Presentations by an authorized administrator of PDXScholar. Please contact us if we can make this document more accessible: pdxscholar@pdx.edu. 
Proximal Predictors of Alcohol Use among Japanese College Students

Staci Wendt, Ph.D.

Research Associate

WestEd

Cynthia Mohr, Ph.D.

Professor

Department of Psychology

Portland State University

Mo Wang, Ph.D.

Professor

University of Florida, Warrington College of Business Administration

Sarah Haverly, B.A.

Graduate Student

Department of Psychology

Portland State University

Running head: Japanese College Student Drinking

This research was supported by grants from the Medical Research Foundation of Oregon and Portland State University. We gratefully acknowledge the assistance of Mika Maruyama and Sarah Paquette. Correspondence regarding this article should be addressed to Cynthia D. Mohr, Department of Psychology, Portland State University, P.O. Box 751, Portland, OR, 97207. Email: cdmohr@pdx.edu. Phone: 503-725-3981, Fax: 503-725-3904. 
Abstract
Background and Objectives: This study investigated how negative social interactions (e.g., disagreeing with a friend) predicted subsequent drinking behaviors among Japanese college students. Because of social influences on drinking, and cultural norms for maintaining social harmony and making amends in response to social transgressions in Japanese culture, the authors hypothesized that students would consume more alcohol socially following increases in negative social interactions. Drinking refusal selfefficacy and social self-efficacy were also studied as moderators of social drinking. Methods: Fifty-five college students (79\% women) of legal drinking age completed a once-daily Internet survey for 30 days, providing 1195 daily reports of drinking and social interaction. Prior to the daily survey, participants reported on Drinking Refusal Self-Efficacy and Social Self-Efficacy in an initial Internet-based assessment. Results: Students drank more socially in the evening following daytime increases in negative social interactions, relative to evenings following fewer such exchanges $(b=.23, p<.001)$. At the betweenperson level, students who reported stronger confidence in refusing to drink drank less socially compared to those who reported less confidence in drinking refusal $(b=-.53, p<.001)$. Yet, those with higher social self-efficacy, which is typically a health-protective factor, drank more socially compared to their counterparts $(b=.32, p<.05)$. Conclusions and Importance: Japanese college students increased their social drinking in response to daily negative social interactions, consistent with the notion that this drinking pattern represents efforts to make amends to others. Interventions targeted toward increasing students' confidence in refusing to drink may be beneficial in reducing social drinking in this population.

Key words: Japan, College student drinking, Daily process methodology, Social Drinking, Self-efficacy 
Alcohol use in Japan has risen dramatically since World War II (Higuchi, Matsushita, \& Osaki, 2006). Findings from the GENACIS (Gender, Alcohol, and Culture: An International Study; Bloomfield et al., 2005) project reveal that, among 23 countries studied, Japan ranked sixth in the number of current drinkers, with approximately $80 \%$ of women and $95 \%$ of men being current drinkers. These findings reflect a dramatic rise from 1968 wherein $76 \%$ of men and $19 \%$ of women reported being current drinkers (Higuchi et al., 2006). A 2013 nationwide survey of drinking-aged adults revealed that $29.4 \%$ of Japanese men and $7.3 \%$ of Japanese women engaged in daily drinking, with $18.6 \%$ of men and $7.4 \%$ of women being categorized as "risky" drinkers (i.e., 280g+ per week for men/168g+ per week for women) (Osaki et al., 2016). These high consumption levels are concerning as Japanese individuals may have biological predispositions to negative health effects from drinking, such as an increased risk of liver disease, and several types of cancer (Luczak, Glatt \& Wall, 2006). This risk may be partly due to the prevalence of the ALDH2 genotype among Japanese persons (Akechi, Iwasaki, Uchitomi, \& Tsugane, 2006), which causes difficulty for the body to clear acetaldehyde, leading to negative health consequences. Japanese drinkers are also at particularly high risk of suicide (Takeshita \& Morimoto, 1999), and report greater prevalence of negative consequences (e.g. feelings of guilt or remorse, failing to do what was normally expected of you) than do their North American and European counterparts (Graham et al., 2011). Indeed, Japanese men are ranked ninth in the world, and women seventh, in the reported experience of negative consequences of drinking (Graham et al., 2011). These factors make it all the more important to understand predictors of alcohol use among this population.

Studies of proximal predictors of drinking among non-Japanese samples have identified the experience of negative social interactions (e.g., having a disagreement with a friend, someone preventing you from reaching your goals) to be a significant risk factor for alcohol use. Indeed, Mohr et al. (2001) found evidence of increases in daily alcohol consumption following the experience of negative social interactions. The relationship between alcohol consumption and negative social interactions is not 
surprising given that such interactions can have the most damaging, long-lasting effects of all stressors (DeLongis, Coyne, Dakof, Folkman \& Lazarus, 1982). Drinking in response to negative social interactions is consistent with the Tension Reduction Hypothesis (Conger, 1956), which posits that individuals consume alcohol to alleviate stress, reinforcing alcohol consumption as a coping mechanism. Tensionreduction drinking is potentially more detrimental than other types of motivated drinking because reliance on alcohol to regulate negative mood may weaken other methods of coping thereby creating a dependency on alcohol (Cooper, Frone, Russell \& Mudar, 1995). Further perceptions that alcohol increases confidence and reduces tension are related to increased alcohol consumption (e.g., Booth \& Hasking, 2009).

The experience of negative social interactions may be especially important to consider in the prediction of drinking in Japan. Research has shown that Japanese individuals are reactive to the emotional experiences and expressions of others, in addition to their own experiences (Kitayama, Markus \& Kurokawa, 2000). Negative social interactions are particularly distressing to Japanese individuals, as they indicate a failure in the task of properly maintaining relationships, as indicated by the experience of shame (Mesquita \& Karasawa, 2002). One recent report indicates that as many as $27.7 \%$ of Japanese men experienced pressure by a spouse or romantic partner to cut down on drinking (within the last 12 months; Hradilova-Selin, Holmila \& Knibbe, 2009). Mesquita and Karasawa (2002) found that Japanese college students reported greater distress in response to thinking about negative interactions with friends, relative to U.S. college students. Failure to maintain social relationships or failing to meet one's social expectations is particularly distressing for the interdependent self and, as a result, causes shame (Benedict, 1946). When individuals experience shame, they seek out others in order to make amends (Markus \& Kitayama, 1991; Mesquita \& Karasawa, 2002). Similarly, after experiencing socially-isolating events, such as a disagreement within one's social group, the 
interdependent self seeks to conform to one's behavior to match other group members (Markus, Kitayama \& Heiman, 1997; Oishi \& Diener, 2001).

Further, social drinking norms have been documented to be relatively strong in Japan (Shimizu, 1990, 2000); drinking with friends ("Tsukiai") is seen as a method to form and affirm connections with one's social group (Shimizu, 2000). Japanese college students also use opportunities to consume alcohol with friends as a method of gaining social capital (McDonald \& Sylvester, 2014) and for deepening friendships (Yamamoto, Saeki, \& Hirano, 2016). Individuals might be at increased risk for heavy alcohol consumption due to the false consensus effect whereby individuals overestimate their friends' drinking behaviors and thus inaccurately adjust their own drinking behaviors to more closely match their perceptions of their friends' drinking behaviors (Ecker, Cohen, \& Buckner, 2017). Additionally, there is a positive relationship between group size and alcohol consumption (Thrul, \& Kuntsche, 2015) and between group size and enhancement-motivated consumption (Thrul \& Kunsche, 2016). Indeed, Shibata (2013) found that Japanese college students' perceptions of peer drinking had the most impact on drinking outcomes, especially for males. In addition to the social aspects of drinking, the college age is an important development time period. Researchers have identified early adulthood as a risky developmental period for excessive alcohol use, typically associated with increased quantity and frequency of consumption (Baer \& Carney, 1993). This developmental period is of concern to health researchers, as habits formed during this period may continue into adulthood (Schulenberg \& Maggs, 2002). Given the strong social influence among Japanese, certain social contexts, such as college, may be risky for young Japanese adults, in terms of pressure to consume alcohol (Higuchi, Matsushita, Muramatsu, Murayama, \& Hayashida, 1996).

Thus, a study investigating the predictors of alcohol consumption for Japanese college student drinkers is needed. Daily process methodology, in which experiences of social interaction and consumption are reported each day as they occur, are uniquely suited to addressing such an inquiry, as 
they provide information regarding intra-individual variation in interpersonal interactions and drinking, and also inter-individual differences. Daily process or diary methodology also allows researchers to establish a temporal sequence between negative social interactions and drinking, as data is collected daily over several weeks. Additionally, this method is useful for collecting data that is vulnerable to retrospective bias, such as emotionally-relevant information (Thomas \& Diener, 1990), and is vital to studying contingencies of drinking behavior (such as the link between social events and subsequent consumption), which cannot be reliably understood or recalled retrospectively (Carney, Tennen, Affleck, Del Boca, \& Kranzler, 1998). Yet, no studies have investigated the intra-individual relationships between negative social interactions and subsequent alcohol consumption in Japanese individuals or the extent to which students drink more following times when they have more negative social interactions, compared to times when they have fewer of those interactions. Because of the strong social normative influences regarding alcohol consumption, for maintaining social harmony and making amends in response to social transgressions, the current study hypothesized that individuals would consume more alcohol with friends after experiencing increases in the number of negative social interactions. This hypothesis is counter to the negative interactions and drinking behavior observed among U.S. college students whereby U.S. college students are more likely to drink alone following negative interactions (Mohr et al., 2005).

In addition to considering negative social interactions as a risk factor for increased daily consumption, it is also important to examine protective factors, such as self-efficacy that may limit or reduce drinking. Self-efficacy has been identified in a number of health models as a major contributing factor to health behaviors (e.g., Theory of the Planned Behavior; Ajzen, 1991). Self-efficacy is defined as one's belief in their own ability to succeed in specific situations or at specific tasks (Bandura, 1992). Generally, self-efficacy has been shown to protect individuals against the negative effects of stressful events (Bandura, 1992). 
Specifically, previous research has identified a type of self-efficacy, drinking refusal self-efficacy (DRSE), or the belief about one's ability to abstain from drinking in certain contexts, as an important factor in determining drinking (Oei, Fergusson \& Lee, 1998; Oei, \& Jardim, 2007). DRSE has been shown to buffer the relationship between social norms for drinking nad alcohol consumption whereby the relationship between social norms and drinking is less strong for individuals with high DRSE compared to individuals with low DRSE (Jang, Rimal, \& Cho, 2013). Among U.S. college students DRSE has been shown to predict higher levels of drinking (Foster, Dukes, \& Sartor, 2016) and be a potential gatekeeper to other risk factors for problematic drinking (Gullo, Dawe, Kambouropoulos, Staiger, \& Jackson, 2010). Further, DRSE has been shown to mediate the association between rash impulsiveness and alcohol misuse (Gullo et al. 2010). Thus, DRSE may be particularly important to Japanese college students who are confronted with strong social pressures to consume alcohol.

We hypothesized that individuals with higher DRSE would consume less with others, compared to individuals with lower DRSE. We also hypothesized that individuals with higher DRSE would consume less alcohol following increases in the occurrence of negative social interactions, compared to individuals with lower DRSE.

Furthermore, social self-efficacy (SSE), or one's beliefs about one's ability to initiate and maintain social relationships, has been shown to be a between-person predictor of health behavior for cultures where maintaining relationships is crucial to well-being (Gong \& Fan, 2006). However, SSE has not previously been investigated in relation to alcohol consumption. Although SSE has not previously been studied in relationship to alcohol consumption, the relationship between social enhancement motivations and alcohol consumption is well-documented. For example, Armeli, Conner, Cullum, and Tennen (2010) found that individuals with high levels of social enhancement drinking behaviors were more likely to use alcohol as a means of coping during stressful or anxiety provoking social interactions. Although the study did not specifically include Japanese college students, one could extrapolate that 
given the desire to make amends in social situations following negative social interactions, individuals who have social enhancement drinking behaviors may be more likely to drink in such contexts. Moreover, social and enhancement motives for drinking have commonly been linked to increases in consumption (Cooper, 1994; Lyvers, Hasking, Hani, Rhodes, \& Trew, 2010) and students who socialize also tend to consume more alcohol (Finlay, Ram, Maggs, \& Caldwell, 2012; Ram, Maggs, \& Caldwell, 2012). Thus, we predicted that individuals with higher SSE, compared to those with lower SSE would report greater social drinking, as this is accepted as one way to affirm relationships. Finally, it was hypothesized that SSE would act as a moderator in the relationship between negative social interactions and drinking, such that individuals with higher SSE would drink more with others following the occurrence of negative social interactions compared to those with lower SSE to make amends in their relationship.

Hypotheses

In sum, based on our literature review of previous studies and theory of social relationships and drinking, we posit the following hypotheses:

Hypothesis 1: Japanese college students would consume more alcohol with friends after experiencing increases in the number of negative social interactions.

Hypothesis 2: Students with higher DRSE would consume less with others, compared to individuals with lower DRSE.

Hypothesis 3: Students with higher DRSE would consume less alcohol following increases in the occurrence of negative social interactions, compared to individuals with lower DRSE.

Hypothesis 4: Students with higher SSE, compared to those with lower SSE would report greater social drinking. 
Hypothesis 5: SSE would moderate the relationship between negative social interactions and drinking, such that students with higher SSE would drink more with others following the occurrence of negative social interactions compared to those with lower SSE.

\section{Method}

\section{Participants}

A convenience sample of fifty-seven Japanese college students ( $79 \%$ women) participated in the daily study of adult health behavior. All participants were enrolled in full-time studies at Waseda University and were recruited both on campus in Japan (28\%) and while participating in an international exchange program in the U.S. (72\%). Students were recruited through academic contacts and email broadcasts; interested students attended a brief orientation to learn more about the study. Our recruitment sample size was determined based on number of observations required for multilevel modeling analyses, as described below. Every student that attended the orientation participated in the study. Students were eligible if they were of legal drinking age (20 years old in Japan and 21 years old in the U.S.) and were full-time college students; the average age of participants was $23(S D=2.96)$ years. One purpose of the study was to examine cross-cultural adjustment, by comparing groups of students in Japan with those temporarily studying in the U.S. However, no such differences were detected; therefore, the student groups were combined for purposes of analysis. The lack of significant findings between the student groups is not surprising, as students studying abroad mostly interacted with other Japanese students studying abroad; integration into U.S. culture was not a primary program focus. Participants received monetary compensation based on the completeness of their surveys. The protocol for this research project was approved by the university's IRB.

\section{Procedure}

After attending the orientation, participants completed the informed consent procedure, followed by an online questionnaire, including SSE and DRSE. Participants were allowed one week to 
complete at baseline. All measures and the informed consent procedure were presented to participants in Japanese. To ensure reliability of translation, all scales were initially translated from English into Japanese and then back-translated by a second translator. The original and the back-translated measures reached high agreement (greater than 93\% agreement for all measures).

\section{Measures}

The 19-item Drinking Refusal Self-Efficacy Questionnaire (DRSE; Oei, Hasking \& Young, 2005) measured individuals' ability to decline an alcoholic beverage in certain circumstances. Participants indicated how sure or unsure they were that they would drink in 19 different situations. There are three subscales: social pressure, emotional relief, and opportunistic drinking. Situations included, e.g., "When I come home from school," "When I am at dinner," and "When someone offers me a drink." The responses range from 1 (I am very sure I would drink) to 6 (I am sure that I would not drink). Higher scores indicate a greater ability to refuse drinking in certain situations. In this sample, a single-factor was found and internal consistency was good $(\alpha=.94 ; M=4.07, S D=.90)$.

The Social Self-Efficacy Questionnaire (SSE; Gong \& Fan, 2006) measured individuals' social selfefficacy. This eight-item scale measures individuals' beliefs about their confidence that they could properly engage in social situations ( $\alpha=.88 ; M=2.82, S D=.77$ ). Participants rated their confidence on a scale of 1 (No confidence) to 5 (Complete confidence). Example items include "Ask a group of people who are planning to engage in a social activity (e.g., to go to a movie) if you can join," and "Get invited to a party that is being given by a prominent or popular individual."

\section{Daily Electronic Survey Procedure}

After completing the initial assessment, participants were sent a link to the daily online survey each day for 30 days. Participants started the daily portion of the study on the same day of the week, but at varying times throughout the year. Participants were sent daily email reminders to complete the survey and were able to access the survey between 3-7p.m. each day. This time period was chosen to 
coincide with the end of a student's workday and beginning of the evening (where drinking is more likely to occur; Mohr et al., 2005). Once a survey was submitted, participants were unable to go back to the survey and change their answers, thus eliminating back-filling of surveys. Participants were asked to report daily negative social interactions that occurred from the time they woke up until the time that they completed the daily survey, as well as drinking from the previous night (i.e., after they completed the diary until they went to bed; Mohr et al., 2005; Wang, Liu, Zhan \& Shi, 2010).

Negative Social Interactions. Negative social interactions were measured using an adapted 14item daily event checklist (Gable, Reis \& Elliot, 2000). Participants were asked to indicate whether events from a checklist occurred that day (e.g., Had a disagreement or conflict with friend(s) or girlfriend/boyfriend,"); if so, participants were asked to rate how desirable the event was on a scale ranging from 1 (extremely undesirable) to 7 (extremely desirable). Events that were appraised as 1-3 on the scale were considered negative events when creating summary scores.

Alcohol Consumption (DRINK). Participants reported the previous night's consumption each day, by reporting the total quantity of alcohol they consumed at home (e.g., in their dorm room) or away from home. Specifically, participants reported how many drinks (between 1 and 15 drinks, > 15 standard drinks; Wechsler et al., 2002) they had at home while drinking alone and how many drinks they consumed while at home drinking with others. Similarly, participants reported how many drinks they consumed away from home alone and away from home with others. Daily consumption was calculated by summing the total number of drinks each individual drank with others (either away from home or at home).

Analytic Strategy

The data were hierarchically structured with 30 possible reports of negative social interactions and DRINKs for each of the 57 participants. Our estimated required sample size was based on guidelines offered by Kreft and DeLeeuw (1998), which summarizes simulation studies that have documented 
power estimates for detecting cross-level moderation. They provide the example of studies with 30 level 2 units with 30 observations, 900 observations total, would render adequate power, defined as .80, for detecting small to moderate effect sizes. Inspection of our dataset revealed that 49 of the 57 provided useable daily data. In particular, of the potential 1470 daily observations (49 people $x 30$ days), $73 \%$ or 1067 daily reports were completed. This compliance rate is comparable to other daily studies of alcohol use (e.g., Mohr et al., 2005). When lagging the negative social interaction predictors to estimate how today's negative interactions predict tonight's drinking (as reported tomorrow), additional data is lost, resulting in 882 observations. This number is consistent with previously published studies examining cross-level interactions of negative experiences and drinking similar to the ones proposed in this study (i.e., Hypotheses 3 and 5; e.g., Liu, Wang, Zhan, \& Shi, 2009). Two additional participants were missing data for the between-person data, rendering a between-person analysis sample of 47.

HLM v6.0 (Raudenbush, Bryk, Cheong \& Congdon, 2004), which allows for unbalanced, hierarchically structured data was used for the analysis. Two equations were specified; a within-person regression model (Level-1 variables) and a between-person regression model (Level-2 variables). The within-person equation included a within-person outcome, which was modeled as a function of the Level-1 predictors. The between-person equation included the intercepts and slopes from Level-1 as a function of the between-person predictors (i.e., DRSE and SSE). Day-of-the-week trends were modeled using six orthogonal dummy variables (Argeriou, 1975; Carney et al., 1998), holding Tuesday constant (Argeriou, 1975; Armeli et al., 2005). Because DRINKs is a count variable, and includes a larger proportion of zeros, a zero-inflated Poisson distribution with a log-link function was employed (Armeli et al., 2005; Raudenbush \& Bryk, 2002). The number of negative social interactions was person-centered (Enders \& Tofighi, 2007); DRSE and SSE were grand-mean centered (Hoffman, 1998). The following equation shows the model used to examine Hypothesis 3 (a moderation equation). Similar equations were used to examine the other hypotheses. 


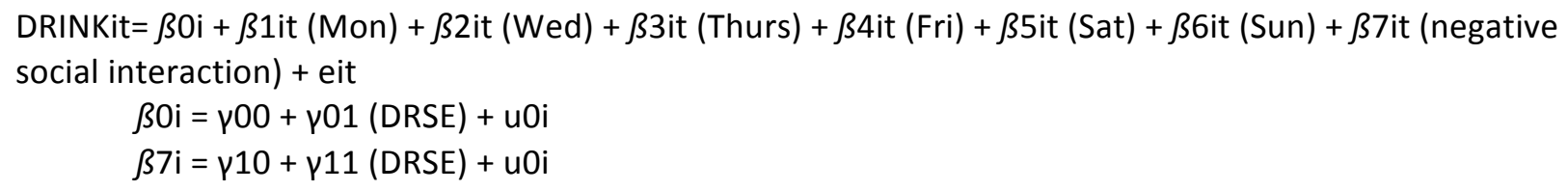

Results

\section{Descriptive Statistics}

Of the final analysis sample of 47 participants, 9 were men (19\%) and 38 were women (81\%). The male participants were, on average, 22.2 years of age $(S D=1.6)$, and the female participants were 23.5 years of age $(S D=3.3)$. Participants consumed alcohol with others on approximately five days throughout the 30-day study ( $M=5.13$ drinks, $S D=3.72)$. On days when participants reported drinking alcohol with others, participants drank almost five drinks $(M=4.65$ drinks, $S D=3.70)$. Participants reported experiencing an average of $1.27(S D=2.51)$ negative social interactions per day across the study.

Hypothesis testing

As hypothesized (see Hypothesis 1), a positive relationship was found between negative social interactions and DRINKs $\left(\beta_{1 i t}=.23\right.$; see Table 1$)$, such that on days when individuals experienced more negative social interactions, relative to days when they experienced fewer negative social interactions, individuals reported greater DRINKs with others. For every one-unit increase in negative social interactions, there was a $26 \%$ increase in the number of DRINKs consumed with others.

As posited in Hypothesis 2, DRSE was examined as a direct predictor of DRINKs, revealing a significant and negative relationship as predicted $\left(\nu_{01}=-.53, p<.001\right.$; see Table 2$)$. For every one-unit increase in DRSE, DRINKs decreased by an average of .58 drinks. Next, the Level-1 intercepts and slopes were modeled as a function of the between-person factor of DRSE (bottom Table 2), revealing that DRSE was a marginally significant moderator of the negative social interactions-DRINKs relationship $\left(\gamma_{11}=-.09\right.$, $p=.08)$. That is, following the occurrence of an increase in negative social interactions, individuals with 
higher DRSE drank marginally less with others compared to those with lower DRSE. Thus, Hypothesis 3 was partially confirmed.

Next, as stated in Hypothesis 4, SSE was investigated as a direct predictor of DRINKs; specifically, the Level-1 intercepts and slopes are modeled as function of the between-person factor of SSE (see Table 3$)$. The results indicated a significant and positive relationship between SSE and DRINKs $\left(\gamma_{01}=.32\right.$, $p<.05)$. Thus, individuals with higher SSE reported greater DRINKs with others, relative to individuals with lower SSE. In the next step, the Level-1 intercepts and slopes were modeled as a function of the level 2 between-person moderator of SSE. Results revealed that SSE did not moderate the negative social interactions-DRINKs relationship $\left(\gamma_{11}=-.06, p=.21\right)$. Thus, Hypothesis 5 was disconfirmed.

\section{Discussion}

Despite relatively high levels of alcohol consumption documented among Japanese individuals, specifically in social contexts (Higuchi et al., 2006), few studies have investigated the proximal predictors of alcohol consumption in this population. The purpose of the current study was to employ daily process methodology to investigate three possible predictors of alcohol use among Japanese college students, including negative social interactions, as well as protective factors of DRSE and SSE, revealing supportive evidence for each hypothesized predictor.

Specifically, results revealed that on days when individuals experienced more negative social interactions, compared to their typical number of negative social interactions, individuals drank more with others. This result is consistent with the interpretation that Japanese college students use drinking with others as a social context to make amends for previous disagreements or failure to maintain social relationships.

Two types of self-efficacy, DRSE and SSE, were investigated as interpersonal predictors of alcohol use, revealing significant results for both. Consistent with previous research, DRSE negatively and significantly predicted social drinking. This finding is consistent with previous studies investigating 
the relationship among U.S. college students, which found that individuals with low DRSE levels have the highest risk of problematic drinking (Foster, Dukes, \& Sartor, 2016). In contrast, SSE positively and significantly predicted social drinking. Recall that SSE is an individual's belief about their ability to engage in social tasks to either initiate or maintain relationships (Smith \& Betz, 2000). Research has shown that individuals with higher SSE feel more confident addressing interpersonal problems and utilize more effective strategies to make amends, compared to individuals with lower SSE (Bilgin \& Akkapulu, 2007). These findings are consistent with the understanding that drinking with others is an important social activity and a way to interact with others in Japan (Shimizu, 1990; 2000).

Our hypothesis that the negative social interactions- social drinking relationship would be weaker for individuals with higher compared to lower DRSE was not supported, although the relationship was marginally significant and consistent with prediction. Further, the moderation effect of SSE on negative social interactions-social drinking was not significant. Although this study included multiple daily measurements of negative social interactions and drinking with others, there was only one measurement of self-efficacy, and the total number of participants was low potentially limiting power. Yet, Bandura $(1986,1997)$ suggested that self-efficacy fluctuates based on contextual influences, affect, and experience; fluctuations that were not captured by the single measurement, suggesting repeated measures of DRSE and SSE may be appropriate. Specific to DRSE, drinking context (e.g., at home or at a bar) is an important predictor of students' DRSE; DRSE is higher in non-alcohol related settings compared to alcohol-related settings (Monk \& Heim, 2013; Bonar, Rosenberg, Hoffman, et al., 2011).

Alternately, if the cultural model dictates that drinking with others is beneficial to relationship well-being and should follow negative interactions, drinking refusal may not be considered culturally appropriate and therefore might not vary significantly across individuals in this context. Further, DRSE may vary for individuals depending on the extent to which alcohol is viewed as central to oneself; Foster 
(2014) found that drinking identity was a mediator between drinking motivations and alcohol consumption. The relationship between DRSE and drinking identity has not previously been studied, but it could be posited that DRSE would be lower for individuals with a stronger drinking identity; future studies should investigate this possible connection.

Our results revealed that higher SSE individuals drank more socially and experienced more negative social interactions compared to their lower SSE counterparts (given a significant and positive correlation was found between negative social interactions and SSE; $r=.39, p<.01)$. Yet, they did not engage in more negative social interactions-related social drinking. Individuals with higher SSE may be more critical of their relationships with others, or more sensitive to negative interactions, which is consistent with research documenting the relative importance of self-criticism within Japanese culture (Kitayama \& Markus, 1999). However, they may respond to negative social interactions in ways other than social drinking. Additionally, there might be an important connection between SSE and social anxiety whereby individuals who have high social anxiety may be more inclined to use alcohol as a social lubricant compared to those who have lower social anxiety (O’Grady, Cullum, Tennen, \& Armeli; 2011). Thus, future research should investigate the role social anxiety may play in the SSE-drinking relationship. Despite study strengths, limitations exist, particularly the relatively large percentage of women in the current sample. Yet, this is an important demographic to consider as researchers have documented a greater increase in the number of reported female drinkers. Specifically, the prevalence of female individuals with alcohol-related problems has increased in recent years, more than doubling from 1984 to 2003 (Osaki et al., 2005). Moreover, Takano, Nakamura and Watanabe (1996) found alcohol consumption to be most prevalent among women ages 20-39 (compared to other age groups of women). Our sample of women college students was 23 years of age, on average, which is comparable to the Takano et al. sample. Although research has focused on many college students "maturing out" of heavy drinking (e.g., Donovan, Jessor \& Jessor, 1983), this may be less true of women graduating college 
and entering the workforce in Japan. In particular, women are at increased risk for developing or maintaining patterns of heavy drinking, as modernist influences encourage women to drink with their professional colleagues, similar to the behaviors traditionally documented among Japanese businessmen (Higuchi et al., 2006).

The current study utilized a convenience sample, such that individuals who participated in the study might not be representative of other Japanese college students. It is important to note that the current study was a part of a larger study on cross-cultural adjustment and health. The original intent was to examine how Japanese students who visited the U.S. as part of a foreign exchange program compared in regards to stress and health with Japanese students from the same university but who remained in Japan. We did not detect significant differences between the two groups and thus proceeded to examine students together as one sample, regardless of the recruitment context. Further, questions regarding consumption were dispersed within other health-related questions, including tobacco and physical health symptoms. Thus, as participants were unaware of the focus on alcohol consumption, it is unlikely that the students who participated in the current study were more or less likely than their peers to consume alcohol.

Despite limitations, the current study makes several notable contributions with important implications. For universities, where there are high levels of alcohol consumption (Baer, 2002), our results indicate that drinking with others serves a purpose in helping Japanese college students maintain social relationships; an important goal of the relatively more interdependent self. Moreover, the results of this study provide support for tailoring interventions to address the unique cultural antecedents of drinking. Specifically, the evidence that individuals in this study drank with others in response to negative social interactions differs from U.S. models that show increases in solitary drinking on days with higher negative social interactions among college students (Mohr et al., 2005). 
Thus, interventions that simply seek to eliminate opportunities where students can engage in drinking with others may be limited in their scope, and have potential negative effects on students' social relationships. In conjunction with the negative significant relationship found between DRSE and drinking with others, college student drinking interventions should focus on providing contexts where alcohol plays a secondary role in activities, or where students feel able to drink less. To better inform intervention efforts, future studies should investigate other methods that Japanese college students utilize to maintain social relationships and to make amends.

In conclusion, this study employed daily process methodology to examine day-to-day variations in alcohol consumption and proximal predictors of alcohol consumption among a Japanese sample. Results revealed an important relationship between daily negative social interactions and subsequent drinking with others. Further, this study was the first to document social self-efficacy as a positive and significant predictor of drinking with others. It also affirmed previous evidence from non-Japanese samples supporting drinking refusal self-efficacy as a significant protective factor for drinking with others. 


\section{References}

Ajzen, I. (1991). The theory of planned behavior. Oganizational Behavior and Human Decision Processes, 50, 179-211.

Akechi, T., Iwasaki, M., Uchitomi, Y., \& Tsugane, S. (2006). Alcohol consumption and suicide among middle-aged men in Japan. The British Journal of Psychiatry, 188, 231-236.

Argeriou, M. (1975). Daily alcohol consumption patterns in Boston: Some findings and a partial test of the Tuesday hypothesis, Journal of Studies on Alcohol, 36, 1578-1583.

Armeli, S., Conner, T. S., Cullum, J., \& Tennen, H. (2010). A longitudinal analysis of drinking motives moderating the negative affect-drinking association among college students. Psychology of Addictive Behaviors, 24(1), 38.

Armeli, S., Mohr, C., Todd, M., Maltby, N., Tennen, H., Carney, M. A., et al. (2005). Daily evaluation of anticipated outcomes from alcohol use among college students. Journal of Social and Clinical Psychology, 24, 767-792.

Baer, J. (2002). Student factors: Understanding individual variation in college drinking. Journal of Studies on Alcohol, Supplement No. 14, 40-53.

Baer, J., \& Carney, M. (1993). Biases in the perceptions of the consequences of alcohol use among college students. Journal of Studies on Alcohol, 54, 54-60.

Bandura, A. (1997). Self-efficacy: The exercise of control. New York: Freeman.

Bandura, A. (1992). Self-efficacy mechanism in psychobiologic functioning. In R. Schwarzer, Selfefficacy: Thought control of action (pp. 355-394). Washington, D.C.: Hemisphere.

Bandura, A. (1986). Social foundations of thought and action: A social cognitive theory. Englewood Cliffs, NJ: Prentice-Hall.

Benedict, R. (1946). The chrysanthemum and the sword: Patterns of Japanese culture. Boston: Houghton-Mifflin. 
Bilgin, M., \& Akkapulu, E. (2007). Some variables predicting social self-efficacy expectation. Social Behavior and Personality, 35, 777-7888.

Bloomfield, K., Allamani, A., Beck, F., Helmersson-Bergmark, K., Csemy, L., Eisenback-Stangl, I., et al. (2005). Gender, culture, and alcohol problems: A multi-national study. Project Final Report. Institute for Medical Informatics, Biometrics, \& Epidemiology. Berlin: Charite Universitatsmedizin.

Bonar, E. E., Rosenberg, H., Hoffmann, E., Kraus, S. W., Kryszak, E., Young, K. M., ... \& Bannon, E. E. (2011). Measuring university students' self-efficacy to use drinking self-control strategies. Psychology of addictive behaviors, 25(1), 155.

Booth, C., \& Hasking, P. (2009). Social anxiety and alcohol consumption: The role of alcohol expectancies and reward sensitivity. Addictive behaviors, 34(9), 730-736.

Carney, M. A., Tennen, H., Affleck, G., Del Boca, F. K., \& Kranzler, H. R. (1998). Levels and patterns of alcohol consumption using timeline follow-back, daily diaries, and real-time "electronic interviews", Journal of Studies on Alcohol, 59, 447-454.

Conger, J.J. (1956). Alcoholism: Theory, problem, and challenge: Reinforcement theory and the dynamics of alcoholism, Quarterly Journal of Studies on Alcohol, 17, 296-305.

Cooper, M.L. (1994). Motivations for alcohol use among adolescents: Development and validation of a four-factor model, Psychological Assessment, 6(2), 117-128.

Cooper, M. L., Frone, M. R., Russell, M., \& Mudar, P. (1995). Drinking to regulate positive and negative emotions: A motivational model of alcohol use. Journal of Personlaity and Social Psychology, 69, 990-1005.

DeLongis, A., Coyne, J.C., Dakof, G., Folkman, S., \& Lazarus, R.S. (1982). Relationship of daily hassles, uplifts, and major life events to health status. Health Psychology, 1(2), 119-136. 
Donovan, J. E., Jessor, R., \& Jessor, L. (1983). Problem drinking in adolescence and young adulthood: A follow-up study. Journal of Studies on Alcohol, 44, 109-137.

Ecker, A. H., Cohen, A. S., \& Buckner, J. D. (2017). Overestimation of close friend drinking problems in the prediction of one's own drinking problems. Addictive behaviors, 64, 107-110.

Enders, C. K., \& Tofighi, D. (2007). Centering predictor variables in cross-sectional multilevel models: A new look at an old issue. Psychological Methods, 12, 121-138.

Finlay, A. K., Ram, N., Maggs, J. L., \& Caldwell, L. L. (2012). Leisure activities, the social weekend, and alcohol use: Evidence from a daily study of first-year college students. Journal of studies on alcohol and drugs, 73(2), 250-259.

Foster, D. W. (2014). Drinking identity as a mediator of the relationship between drinking motives and weekly alcohol consumption among heavy drinking undergraduate students. Addictive behaviors, 39(12), 1811-1815.

Foster, D. W., Dukes, K., \& Sartor, C. E. (2016). The road to drink is paved with high intentions: Expectancies, refusal self-efficacy, and intentions among heavy drinking college students. Alcohol, 50, 65-71.

Gable, S., Reis, H., \& Elliot, A. J. (2000). Behavioral activation and inhibition in everyday life. Journal of Personality and Social Psychology, 78, 1135-1149.

Gong, Y., \& Fan, J. (2006). Longitudinal examination of the role of goal orientation in cross-cultural adjustment. Journal of Applied Psychology, 91, 176-184.

Graham, K., Bernards, S., Knibbe, R., Kairouz, S., Kuntsche, S., Wilsnack, S. C., ... \& Gmel, G. (2011). Alcohol-related negative consequences among drinkers around the world. Addiction, 106(8), 1391-1405. 
Gullo, M. J., Dawe, S., Kambouropoulos, N., Staiger, P. K., \& Jackson, C. J. (2010). Alcohol expectancies and drinking refusal self-efficacy mediate the association of impulsivity with alcohol misuse. Alcoholism: Clinical and Experimental Research, 34(8), 1386-1399.

Higuchi, S., Matsushita, S., \& Osaki, Y. (2006). Drinking practices, alcohol policy and prevention programs in Japan, International Journal of Drug Policy, 102, 358-366.

Higuchi, S., Matsushita, S., Muramatsu, T., Murayama, M., \& Hayashida, M. (1996). Alcohol and aldehyde dehydrogenase genotypes and drinking behavior in Japanese, Journal of Clinical and Experimental Research, 20, 493-497.

Hoffman, D. (1998). Centering decisions in hierarchical linear models: Implications for research in organizations. Journal of Management, 24, 623-641.

Hradilova-Selin, K., Holmila, M., \& Knibbe, R. A. (2009). Informal social control of drinking in intimate relationships-a comparative analysis. Contemporary drug problems, 36(1-2), 31-58.

Jang, S. A., Rimal, R. N., \& Cho, N. (2013). Normative influences and alcohol consumption: the role of drinking refusal self-efficacy. Health communication, 28(5), 443-451.

Kitayama, S., \& Markus, H. R. (1999). Yin and yang of the Japanese self: The cultural psychology of personality coherence. In D. Cervone, \& Y. Shoda, The coherence of personality: Social cognitive bases of personality consistency, variability, and organization (pp. 242-302). New York: Guilford Press.

Kitayama, S., Markus, H. R., \& Kurokawa, M. (2000). Culture, emotion, and well-being: Good feelings in Japan and the United States. Cognition and Emotion, 14, 93-124.

Kreft, I. G., \& de Leeuw, J. (1998). Introducing multilevel modeling. London, UK: Sage.

Liu, S., Wang, M., Zhan, Y., \& Shi, J. (2009). Daily work stress and alcohol use: testing the cross-level moderation effects of neuroticism and job involvement. Personnel Psychology, 62(3), 575-597.

Luczak, S. E., Glatt, S. J., \& Wall, T. L. (2006). Meta-analyses of ALDH2 and ADH1B and alcohol 
dependence in Asians: Examining models of influence, Psychological Bulletin, 132, 607-621.

Lyvers, M., Hasking, P., Hani, R., Rhodes, M., \& Trew, E. (2010). Drinking motives, drinking restraint and drinking behaviour among young adults. Addictive behaviors, 35(2), 116-122.

Markus, H. R., \& Kitayama, S. (1991). Culture and the self: Implications for cognition, emotion, and motivation. Psychological Review, 98, 224-253.

Markus, H. R., Kitayama, S., \& Heiman, R. (1997). Culture and "basic" psychological principles. In E. T. Higgins, \& A. W. Kruglanski, Social psychology: Handbook of basic principles (pp. 857-913). New York: Guilford Press.

McDonald, B., \& Sylvester, K. (2014). Learning to get drunk: The importance of drinking in Japanese university sports clubs. International review for the sociology of sport, 49(3-4), 331-345.

Mesquita, B., \& Karasawa, M. (2002). Different emotional lives. Cognition and Emotion, 16, 127-141.

Mohr, C. D., Armeli, S., Tennen, H., Carney, M. A., Affleck, G., \& Hromi, A. (2001). Daily interpersonal experiences, context, and alcohol consumption: Crying in your beer and toasting to good times. Journal of Personality and Social Psychology, 80(3), 489-500.

Mohr, C. D., Armeli, S., Tennen, H., Temple, M., Todd, M., Clark, J., et al. (2005). Moving beyond the key party: A daily process investigation of college student drinking motivations. Psychology of Addictive Behaviors, 34, 222-254.

Monk, R. L., \& Heim, D. (2013). Environmental context effects on alcohol-related outcome expectancies, efficacy, and norms: a field study. Psychology of Addictive Behaviors, 27(3), 814.

Oei, T. P., \& Jardim, C. L. (2007). Alcohol expectancies, drinking refusal self-efficacy and drinking behaviour in Asian and Australian students. Drug and alcohol dependence, 87(2), 281-287.

Oei, T. P., Hasking, P. A., \& Young, R. (2005). Drinking refusal self-efficacy questionnaire revised (DRSEQ-R): A new factor strucutre with confirmatory factor analysis. Drug and Alcohol Dependence, 78, 297-307. 
Oei, T., Fergusson, S., \& Lee, N. (1998). The differential role of alcohol expectancies and drinking refusal self-efficacy in problem and nonproblem drinkers. Journal of Studies on Alcohol, 59, 704711.

O'Grady, M. A., Cullum, J., Tennen, H., \& Armeli, S. (2011). Daily relationship between event-specific drinking norms and alcohol use: A four-year longitudinal study. Journal of studies on alcohol and drugs, 72(4), 633-641.

Oishi, S., \& Diener, E. (2001). Goals, culture, and subjective well-being. Personality and Social Psychology Bulletin, 27, 1674-1682.

Osaki, Y., Kinjo, A., Higuchi, S., Matsumoto, H., Yuzuriha, T., Horie, Y., Kimura, M., Kanda, H. \& Yoshimoto, H. (2016). Prevalence and trends in alcohol dependence and alcohol use disorders in japanese adults; results from periodical nationwide surveys. Alcohol and Alcoholism, 51(4), 456473.

Osaki, Y., Matsushita, S., Shirasaka, T., Hiro, H., \& Higuchi, S. (2005). Nationwide survey of alcohol drinking and alcoholism among Japanese adults. Japanese Journal of Alcohol and Drug Dependence, 40, 455-470.

Raudenbush, S. W., \& Bryk, A. S. (2002). Hierarchical linear models: Applications and data analysis methods. (2nd ed.). Newbury Park: Sage.

Raudenbush, S. W., Bryk, A. S., Cheong, Y. F., \& Congdon, R. T. (2004). HLM 6: Hierarchical linear and nonlinar modeling. Chicago: Scientific Software International.

Schulenberg, J.E., \& Maggs, J. L. (2002). A Developmental Perspective on Alcohol Use and Heavy Drinking during Adolescence and the Transition to Young Adulthood. Journal of Studies on Alcohol, Supplement No. $14,54-70$. 
Shibata, Y. (2013). Sex differences in the effects of disinhibition, perceived peer drinking, and delay discounting on drinking among Japanese college students. Personality and Individual Differences, 55(7), 766-770.

Shimizu, H. (2000). Japanese cultural psychology and empathic understanding: Implications for academic and cultural psychology. Ethos, 28, 224-247.

Shimizu, H. (1990). An alcoholic social system: Drinking culture and drinking behaviors in Japan. Japanese Journal of Mental Health, 36, 85-100.

Smith, H., \& Betz, N. (2000). Development and evaluation of a measure of social self-efficacy in college students. Journal of Career Assessment, 56, 35-52.

Takano, T., Nakamura, K., \& Watanabe, M. (1996). Increased female drinking in accordance with posindustrial urbanization in Japan. Alcohol and Alcoholism, 31, 41-49.

Takeshita, T., \& Morimoto, K. (1999). Self-reported alcohol-associated symptoms and drinking behavior in three ALDH2 genotypes among Japanese university students. Alcohol: Clinical and Experimental Research, 23, 1065-1069.

Thomas, D. L., \& Diener, E. (1990). Memory accuracy in the recall of emotions. Journal of Personality and Social Psychology, 59, 291-297.

Thrul, J., \& Kuntsche, E. (2016). Interactions between drinking motives and friends in predicting young adults' alcohol use. Prevention Science, 17(5), 626-635.

Thrul, J., \& Kuntsche, E. (2015). The impact of friends on young adults' drinking over the course of the evening - an event-level analysis. Addiction, 110(4), 619-626.

Wang, M., Liu, S., Zhan, Y., \& Shi, J. (2010). Daily work-family conlflict and alcohol use: Testing the crosslevel moderation effects of peer drinking norms and social support, Jounral of Applied Psychology, 62, 575-597.

Wechsler, H., Nelson, T. F., Lee, J. E., Seibring, M., Lewis, C., \& Keeling, R. P. (2002). Perception and 
reality: A national evaluation of social norms marketing interventions to reduce college students' heavy alcohol use. Quarterly Journal of Studies on Alcohol, 64, 484-494.

Yamamoto, K., Saeki, K., \& Hirano, M. (2016). The Associations of Drinking Behavior with Friendship Relations and Personality Traits among Underage University Students. Japanese Journal of Public Health Nursing. 5(1), 29-36. 
Table 1. NIEs predicting DRINKs with others

\begin{tabular}{|c|c|c|c|}
\hline Variable & Coefficient & SE & $\begin{array}{l}\text { Confidence } \\
\text { Interval }\end{array}$ \\
\hline \multicolumn{4}{|l|}{ Level 1} \\
\hline Intercept & $-1.16 * *$ & 0.45 & $0.13,0.78$ \\
\hline NIEs & $0.23 * * *$ & .04 & $1.16,1.37$ \\
\hline \multicolumn{4}{|l|}{ Variance Components } \\
\hline Intercept & $0.75 * * *$ & & \\
\hline NIEs & $0.05^{*}$ & & \\
\hline
\end{tabular}

Note: Dependent variable is DRINKs. DRINKs=number of drinks with others, NIEs=negative interpersonal exchanges.

${ }^{*} p<.05$

$* * p<.01$

$* * * \mathrm{p}<.001$ 
Table 2. DRSE predicting DRINKs with others

\begin{tabular}{lccc}
\hline \multicolumn{1}{c}{ Variable } & Coefficient & SE & $\begin{array}{c}\text { Confidence } \\
\text { Interval }\end{array}$ \\
\hline $\begin{array}{l}\text { Level } 1 \\
\text { Intercept }\end{array}$ & $-1.23^{* *}$ & 0.44 & $0.12,0.72$ \\
Level 2 & & & \\
DRSE & $-0.53^{* * *}$ & 0.13 & $0.45,0.76$ \\
Variance Components & & & \\
Intercept & $0.54^{* * *}$ & &
\end{tabular}

Model including NIE and DRSE X NIE interaction

Level 1

Intercept

$-1.25 * *$

0.46

$0.11,0.72$

NIEs

$0.23 * * *$

0.05

$1.13,1.41$

Level 2

DRSE

$-0.56^{* * *}$

0.12

$0.44,0.73$

DRSE X NIES

$-0.09$

0.05

$0.83,1.01$

Variance Components

Intercept

$0.54 * * *$

NIES

0.05

Note: Dependent variable is DRINKs. DRINKs=number of drinks with others, NIEs=negative interpersonal exchanges, DRSE=drinking refusal self-efficacy.

\footnotetext{
$* * p<.01$

$* * * p<.001$
} 
Table 3. SSE predicting DRINKs with others

\begin{tabular}{lccc}
\hline \multicolumn{1}{c}{ Variable } & Coefficient & SE & $\begin{array}{c}\text { Confidence } \\
\text { Interval }\end{array}$ \\
\hline $\begin{array}{l}\text { Level } 1 \\
\text { Intercept }\end{array}$ & $-1.15^{* *}$ & 0.42 & $0.13,0.74$ \\
Level 2 & & & \\
SSE & $0.32^{*}$ & 0.15 & $1.02,1.87$ \\
Variance Components \\
Intercept
\end{tabular}

Model including NIE and SSE X NIE interaction

Level 1

$\begin{array}{lccc}\text { Intercept } & -1.17^{* *} & 0.43 & 0.13,0.73 \\ \text { NIEs } & 0.23^{* * *} & 0.03 & 1.18,1.35\end{array}$

Level 2

$\begin{array}{llll}\text { SSE } & 0.37^{* *} & 0.14 & 1.09,1.95 \\ \text { SSE X NIES } & -0.09 & 0.06 & 0.86,1.03 \\ \text { Variance Components } & & & \\ \text { Intercept } & 0.76^{* * *} & & \\ \text { NIEs } & 0.04 & & \end{array}$

Note: Dependent variable is DRINKs. DRINKs=number of drinks with others, SSE=social self-efficacy.

$* p<.05$

$* * p<.01$

$* * * p<.001$ 